\title{
A New Method Based on LAMP-CRISPR-Cas I2a- Lateral Flow Immunochromatographic Strip for Detection
}

\author{
Huaming Xu', Hao Tang ${ }^{2}$, Rongrong $\mathrm{Li}^{3}$, Zhaoxin $\mathrm{Xia}^{2}$, Wensu Yang ${ }^{2}$, Yi Zhu ${ }^{2}$, Zhen Liu ${ }^{2}$, Guoping Lu ${ }^{4}$, \\ Shenwang $\mathrm{Ni}^{2}$, Jilu Shen $\mathbb{D}^{\prime}$ \\ 'Department of Laboratory Medicine, The Fourth Affiliated Hospital of Anhui Medical University, Hefei, 2300I2, People's Republic of China; \\ ${ }^{2}$ University Laboratory, The Fourth Affiliated Hospital of Anhui Medical University, Hefei, 230012, People's Republic of China; ${ }^{3}$ The First Affiliated \\ Hospital of Anhui Medical University Laboratory Department, Hefei, Anhui, Peoples' Republic of China; ${ }^{4}$ Laboratory Department of Fuyang Hospital \\ Affiliated to Anhui Medical University, Fuyang, Anhui, People's Republic of China \\ Correspondence: Jilu Shen, Tel +86 I5I 55I5 2963, Email shenjilu@ahmu.edu.cn; I464675852@qq.com
}

\begin{abstract}
Introduction: Carbapenemase-mediated antimicrobial resistance is currently a hot spot of global concern. Carbapenem-resistant organisms are highly prevalent in hospitals associated with difficult-to-treat infections, resulting in poor clinical outcome due to limited treatment options. It is urgently needed to have a rapid, efficient, and convenient molecular assay for identifying such resistant strains.
\end{abstract}

Methods: For this end, we developed a new laboratory assay targeting Klebsiella pneumoniae carbapenemase (KPC) and New Delhi metallo- $\beta$-lactamase (NDM) based on loop-mediated isothermal amplification, CRISPR-Cas12a, and lateral flow immunochromatographic strip (CRISPR-Cas-LAMP-lateral flow strip). The method was designed to use a guide RNA (gRNA) to recognize the target DNA and guide Cas12a to cleave the target DNA, and simultaneously cleave any single-stranded DNA within the cleavage reaction system.

Results: The cleavage products are visible to the naked eye on the lateral flow strip. This method is highly sensitive in direct detection of bacteria in samples containing at least $3 \times 10^{5} \mathrm{CFU} / \mathrm{mL}$ without the need for bacterial culture.

Discussion: It provides shorter turnaround time and higher specificity than the conventional bacterial culture and susceptibility testing method. This new assay is applicable for extensive use in hospital infection control, as well as identification and treatment of resistant strains due to simple operation and inexpensive apparatuses.

Keywords: carbapenemase, Klebsiella pneumoniae carbapenemase, New Delhi metallo- $\beta$-lactamase, loop-mediated isothermal amplification, CRISPR-Cas12a, lateral flow immunochromatographic strip, detection

\section{Introduction}

About 700,000 people die from "superbugs" globally every year. Antibiotic abuse is the culprit of extensive spread of superbugs. Currently, carbapenemase-producing organisms are among the most important pathogens of hospital infections. Especially the plasmid-mediated highly transmissible carbapenem-resistant Enterobacterales (CRE) has become an important public health issue of global concern.

Carbapenemases are a broad category of beta-lactamases which can hydrolyze nearly all beta-lactam antibiotics, including carbapenems. Carbapenemases are primarily classified into Ambler classes A, B, and D. The infections caused by such carbapenem-resistant organisms usually also resist other commonly used antimicrobial agents, resulting in the dilemma of no effective drug available in clinical practice. Antibiotic-resistant infections are associated with longer hospital stay and higher medical expenses and mortality. Since Klebsiella pneumoniae carbapenemase (KPC)-producing K. pneumoniae was reported for the first time in 2001, KPC-producing Enterobacterales have been spreading extensively, and resulted in outbreaks across the world. It has been reported that carbapenemase-producing Enterobacterales have 
been identified in more than 10 European, American, and Asian countries. However, the lack of a rapid, convenient, and accurate molecular diagnostic assay has hampered the response of public health services to the rapid spread of drugresistant bacteria.

Currently, two categories of laboratory tests (phenotypic assays and molecular tests) are available for detecting carbapenemases in clinical settings. Conventional methods include the disk diffusion method, modified carbapenem inactivation method (mCIM) combined with EDTA-modified carbapenem inactivation test (eCIM), modified Hodge test (MHT), polymerase chain reaction (PCR), and sequencing analysis. These assays are reliable but associated with shortcomings such as a high rate of false-positive results, longer turnaround time (TAT), higher cost, and complex operating procedures. These limitations usually result in delayed laboratory reports of CRE, which not only encourage the clinicians to prescribe expensive antimicrobial therapy empirically, but also discourage clinicians' confidence in the result of the carbapenemase assay. Delay in CRE detection will seriously prevent the success of hospital infection control. It is therefore vitally important to develop an efficient, accurate, convenient, and rapid assay for detection of carbapenemase-producing strains.

Loop-mediated isothermal amplification (LAMP) was invented by Japanese scholar Notomi for in vitro amplification of nucleic acids. This technique requires 4 to 6 specifically designed primers to recognize the unique target DNA fragment. In the presence of strand displacement DNA polymerase, a large number of amplificons can be produced at $65^{\circ} \mathrm{C}$ in a very short time. LAMP is 100-1000 times more efficient than the standard PCR. A water bath is enough equipment for the LAMP reaction, which greatly reduces the cost. The LAMP technique has been used extensively in detection of pathogens. Additionally, some novel techniques for nucleic acid amplification are developed, including recombinase polymerase amplification (RPA), rolling circle amplification (RCA), nucleic acid sequence-based amplification (NASBA), and single primer isothermal amplification (SPIA). These techniques are really valuable in rapid detection of carbapenemases and feasible to some extent, but there are still some false-positive results. The assay-related cross contamination also seriously affects the interpretation of results.

In recent years, the Clustered Regularly Interspaced Short Palindromic Repeats (CRISPR) has become a powerful gene editing tool. ${ }^{1}$ When testing, Cas12a protein is guided by a gRNA to recognize the target sequence complementary to the gRNA, and cleave both the target sequence and any single-stranded DNA in the reaction system. Taking advantage of this feature of CRISPR, we designed a single-stranded DNA labeled with both fluorescein and quencher. The gRNA cannot recognize the target sequence and cleave the single-stranded DNA in the reaction system if the target sequence is absent. The double-labeled single-stranded DNA probe will be cleaved, and the fluorescence value will increase significantly if the target sequence is present. ${ }^{2}$ This method is sensitive and specific, but time-consuming and expensive due to reliance on fluorescent instruments. Under resource-constrained circumstances, it is very inconvenient to use this method.

For the purpose of optimizing the technique, we have tried to develop a novel method based on LAMP-Cas12a-lateral flow immunochromatographic strip, ie, DNA Endonuclease Targeted CRISPR Trans Reporter (DETECTR) for detection of CRE. Specifically, when a target gene is recognized, it can nonspecifically cleave the single-stranded DNA labeled with both fluorescein and biotin. The cleaved probes can be captured by anti-FAM antibodies in the quality control line or goat anti-mouse antibodies in the test line to produce corresponding bands. The probes not cleaved are only captured by the anti-FAM antibodies in the quality control line to display corresponding bands. This method is easily to operate at low cost with high sensitivity and specificity. ${ }^{3}$ The results are visible to the naked eye. It is applicable to any point-ofcare location out of the laboratory. For this reason, it is widely adopted in clinical laboratory practice. ${ }^{4}$ The latest report has confirmed its successful application in detection of SARS-CoV-2. ${ }^{5}$

\section{Materials and Methods}

\section{Reagents and Chemicals}

All the primers (Table 1) used in this study were synthesized by Shanghai Sangon Biotech Company (Shanghai, China). Anti-FAM antibody, goat anti-mouse antibody, and nitrocellulose membrane were purchased from Zoonbio Biotechnology Company. Quencher and fluorescein double-labeled reporter molecule, biotin and quencher doublelabeled reporter molecule, and crRNAs were bought from Shanghai Genebiogist Technology Co, Ltd (Shanghai, 
Table I The Oligonucleotide Sequences Used as in This Study

\begin{tabular}{|l|l|}
\hline Oligonucleotide & Sequence (5'-3') \\
\hline $\begin{array}{l}\text { SSDNA reporters } \\
\text { PCR primers } \\
\text { Forward primer } \\
\text { Reverse primer } \\
\text { LAMP primer }\end{array}$ & FAM-TTATTTTATT-biotin/FAM-TTAAAATATT-BHQ \\
F3 & GACACACCCATCCGTTACG/CATTAGCCGCTGCATTGATG \\
B3 & GAACGTGGTATCGCCGAT/GGCTGCGAGCCAGCACAGC \\
FIP & GGAACGTGGTATCGCCGAT/GGCTGCGAGCCAGCACAGC \\
BIP & CGCCACCGTCATGCCTGTT-CGCTGGTTCCGTGGTCAC \\
CrRNA & ATCGCCAAACCGTTGGTCGCC-CGGTGAAATCCGCCCG \\
Activator & TGGTTTTCCGCCAGCTCGC-AGCGACTGCCCCGAAAC \\
& UAAUUUCUACUAAGUGUAGAUUUGCUGAAGGAGUUGGGCGG \\
& UAAUUUCUACUAAGUGUAGAUCGCCAGCUCGCACCGAAUGU \\
\hline
\end{tabular}

China). WarmStart LAMP Kit, LbaCas12a, NEB buffer 3.1, and WarmStart Bst 2.0 DNA Polymerase were obtained from New England Biolabs (NEB). Deoxynucleoside triphosphate (dNTPs) and Taq polymerase premixture were purchased from Takara (Beijing, China). Chlor-auric acid $\left(\mathrm{HAuCl}_{4} \cdot 3 \mathrm{H}_{2} \mathrm{O}\right)$ and trisodium citrate were obtained from Sigma-Aldrich (Steinheim, Germany). Absorbent paper, glass fiber paper, and nitrocellulose film were purchased from Sartorius AG (Gottingen, Germany). Genomic and plasmid extraction kits were purchased from Qiagen GmbH (Hilden, Germany) and Tiangen Biotech (Beijing, China) Co., Ltd, respectively.

\section{Methods}

\section{Preparation of Colloidal Gold Nanoparticles and Couplers}

Gold chloride solution $\left(\mathrm{HAuCl}_{4}, 350 \mathrm{~mL}, 1 \mathrm{mM}\right)$ was heated to boil. After boiling, stirred the solution and added $3.5 \mathrm{~mL}$ sodium citrate solution (1\%), which made the mixture colorless. The mixture turned to wine red color after boiling for another 10 minutes. The solution was cooled at room temperature for later use. For preparation of AuNPanti-biotin antibody conjugate, colloidal gold nanoparticles were prepared by centrifugation $(21,100 \times \mathrm{g})$ for $25 \mathrm{~min}$ to collect $1 \mathrm{~mL}$ nanoparticles, repeated 4 times, then mixed with $100 \mu \mathrm{L}$ suspension buffer $\left(10 \%\right.$ sucrose, $0.1 \% \mathrm{NaN}_{3}, 5 \%$ BSA, $0.25 \%$ Tween-20, and $20 \mathrm{mM} \mathrm{Na}_{3} \mathrm{PO}_{4}$ ). Finally, added $0.5 \mathrm{mg} / \mathrm{mL}$ anti-biotin antibody, gently shook for $3 \mathrm{~h}$ at 4 ${ }^{\circ} \mathrm{C}$, followed by centrifugation $(21,100 \times \mathrm{g})$ for $25 \mathrm{~min}$, and rinsed with suspension buffer for 3 times to remove the free anti-biotin antibody. The red particles were resuspended in $100 \mu \mathrm{L}$ suspension buffer, and dispensed onto conjugate pad.

\section{Construct DETECTR Lateral Flow Immunochromatographic Strip}

As illustrated, the lateral flow immunochromatographic strip is composed of three main parts: sample pad, binding pad, and nitrocellulose membrane. The anti-FAM antibody and anti-mouse IgG antibody were spread onto the nitrocellulose membrane strip (width $25 \mathrm{~mm}$ ) to form a control area and a test area. The distance between the test line and the control line was kept greater than $5 \mathrm{~mm}$. Test strips were dried at $22{ }^{\circ} \mathrm{C}$ for 12 hours, and stored in a container until use. Desiccant was placed in the container to prevent the unexpected effect of moisture of nitrocellulose membrane on test results. For the purpose to assemble an intact test strip, the sample pad (width $16 \mathrm{~mm}$ ) was immersed in sample pad buffer (1\% Triton, $1 \%$ BSA, $2 \%$ glucose, $50 \mathrm{~mm}$ boric acid, $\mathrm{pH} 8.0$ ), dried, and stored. All the pads were assembled in turn along the adhesive part of the nitrocellulose membrane with $2 \mathrm{~mm}$ overlap, and cut into strips of $0.4 \mathrm{~cm}$ width. The self-prepared sample pads and absorbent pad were soaked in the $\mathrm{pH} 8.0$ buffer containing $1 \%$ Triton, 1\% BSA, 2\% glucose, and $50 \mathrm{mM}$ boric acid, dried and stored in a dry container. The binding pads and absorbent pads were used directly without processing in buffer. They were assembled successively along the nitrocellulose membrane. 


\section{Target DNA Design}

The full-length specific conserved DNA sequences of the genes encoding KPC enzyme (813 bp) and NDM enzyme (813 bp) were retrieved from GenBank database at NCBI for designing the most appropriate primers via the Primer explorer V5 website. The primers were used for LAMP experiments, fluorescence quantitative PCR, and gene editing.

\section{Screening KPC- and NDM-Producing Resistant Strains}

EDTA-modified carbapenem inactivation test (eCIM) was combined with modified carbapenem inactivation method (mCIM) to harvest strains: a full loop of bacteria $(1 \mu \mathrm{L})$ were picked from overnight cultured bacterial colonies on blood plate and put into a tube containing $2 \mathrm{~mL}$ trypticase soy broth (TSB) medium. ${ }^{6}$ The tube was shaken for $10-15$ seconds to mix well. A germ-free meropenem disk was put into each tube to make sure that the disk was completely immersed in bacterial suspension. The suspension was cultured at $37{ }^{\circ} \mathrm{C}$ overnight. E. coli ATCC25922 suspension (0.5 McFarland standard) was prepared in nutrient broth immediately after the end of incubation. Bacterial suspension preparation and plate streaking were completed in 10-15 minutes. ${ }^{7}$ The plates were dried for 3-10 minutes. The bacterial suspension was taken from TSB with an inoculation loop $(10 \mu \mathrm{L})$ to streak E. coli ATCC25922 onto a Mueller Hinton agar (MHA) plate. The plate was inverted to measure the diameter of inhibition zone after incubation. Meanwhile, $20 \mu \mathrm{L}$ of $0.5 \mathrm{M}$ EDTA solution was put into another TSB tube, and diluted to $2 \mathrm{~mL}$ to bring the final EDTA concentration to $5 \mathrm{mM}$. The following procedures were repeated just like in mCIM. Both mCIM and eCIM meropenem disks were placed onto the MHA plate streaked with E. coli ATCC25922. The plates were observed visually after incubation for bacterial growth. ${ }^{8}$ The results are shown in Figure 2.

\section{Bacterial Collection and DNA Extraction}

The carbapenemase-producing K. pneumoniae strains were selected after being confirmed by eCIM and mCIM screening tests. The concentration of bacterial suspension was adjusted to $2.0 \mathrm{McFarland}$ standard, and subject to a metal bath for 8 minutes, then centrifuged at $12,000 \mathrm{rpm}$ for 10 minutes. The supernatant was taken as the target gene. ${ }^{9}$

\section{LAMP and PCR Amplification}

The crude DNA was used as the template for PCR/LAMP amplification and CRISPR-Cas12a reaction. The amplification was performed per the instruction for use of the test kit. The LAMP reaction system included primers F3, B3 $(0.2 \mu \mathrm{M})$, FIB, FIP $(1.6 \mu \mathrm{M}), 1.4 \mathrm{mM}$ dNTP, $6 \mathrm{mM} \mathrm{MgSO}_{4}, 1 \times$ isothermal amplification buffer, $320 \mathrm{U} / \mathrm{mL}$ WarmStart Bst 2.0 DNA polymerase, and $2 \mu \mathrm{L}$ target DNA template. $\mathrm{ddH}_{2} \mathrm{O}$ was added to bring the final volume to $25 \mu \mathrm{L}$. The amplification was run at $65{ }^{\circ} \mathrm{C}$ for 40 minutes on a fluorescence quantitative PCR instrument. ${ }^{10}$ The amplified products were observed by agarose gel electrophoresis.

Conventional PCR reaction system is composed of $50 \mu \mathrm{L} 1 \times$ rTaq premixture, $1 \mu \mathrm{L}$ forward and reverse primers $(0.5$ $\mu \mathrm{M}), 1-2 \mu \mathrm{L}$ genomic DNA (200 ng), and $\mathrm{ddH}_{2} \mathrm{O}$. The final reaction volume was $50 \mu \mathrm{L} .{ }^{11}$ The reaction included denaturation at $95{ }^{\circ} \mathrm{C}$ for 5 minutes, followed by 35 cycles of reaction (ie, denaturation at $95{ }^{\circ} \mathrm{C}$ for 10 seconds, annealing at $55^{\circ} \mathrm{C}$ for 10 seconds, extension at $72{ }^{\circ} \mathrm{C}$ for 30 seconds). Some fluorescence and gel results of PCR and lamp are shown in Figure 3.

\section{Cas I 2a Trans-Cleavage Assay}

Cas12a cleavage reaction system $(25 \mu \mathrm{L})$ is composed of $5 \mu \mathrm{L}$ NEB 3.1 buffer, $3 \mu \mathrm{L}$ crRNA $(10 \mu \mathrm{M}), 1 \mu \mathrm{L}$ LbCas12a $(40 \mathrm{nM}), 1 \mu \mathrm{L}$ FAM-biotinylated reporter molecule $(60 \mathrm{nM})$, and LAMP amplification products $(3 \mu \mathrm{L}) . \mathrm{ddH}_{2} \mathrm{O}(12 \mu \mathrm{L})$ was added to bring the final volume to $25 \mu \mathrm{L} .{ }^{12}$ The mixture was then incubated at $37{ }^{\circ} \mathrm{C}$ for 30 minutes, mixed once every 10 minutes. Diluent was added appropriately dependent on the target concentration. The supernatant $(80 \mu \mathrm{L})$ was slowly added into the holes of the test strip. The results were interpreted 7-10 minutes later (Figure 1). The result is positive if a band appears on the test line or on both the control line and the test line. ${ }^{13}$ The result is negative if a band appears on the control line alone (see Figure 4).

As described above, for fluorescence analysis of trans-cleavage reaction, 5'-FAM- and 3'-BHQ-labeled ssDNA reporter molecule was used to replace FAM fluorescein and biotin-labeled ssDNA reporter molecule to prepare $25 \mu \mathrm{L}$ reaction system. ${ }^{14}$ The reaction was incubated and run at $37{ }^{\circ} \mathrm{C}$ in the Thermo Fisher 7500 Quantitative PCR System. 

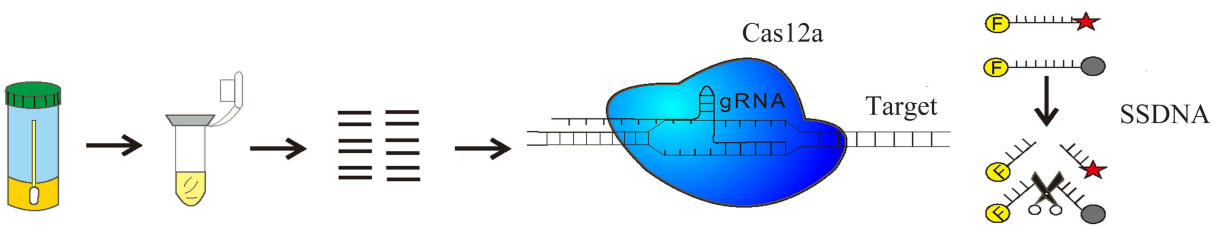

(B)

FAM fluorescein $\star$

BHQ quencher

Biotin

Clinical specimen DNA extraction LAMP amplification Cas12a cleavage reaction collection

probe reporter cleavage
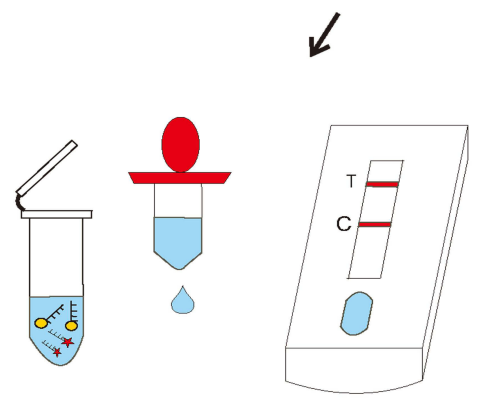

Detection of SSDNA reporter

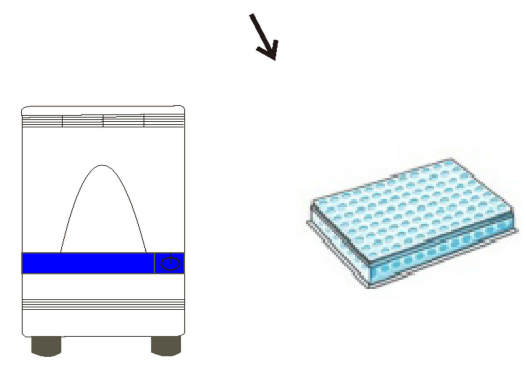

Fluorescence detection by fluorescence quantitative PCR

Figure I The nucleic acid detection platform based on CRISPR-Cas gene editing technology can be used either in laboratories equipped with complex instruments for large volume detection, or in the resource-constrained point of care environment for rapid detection by lateral flow immunochromatographic strip within about I hour.

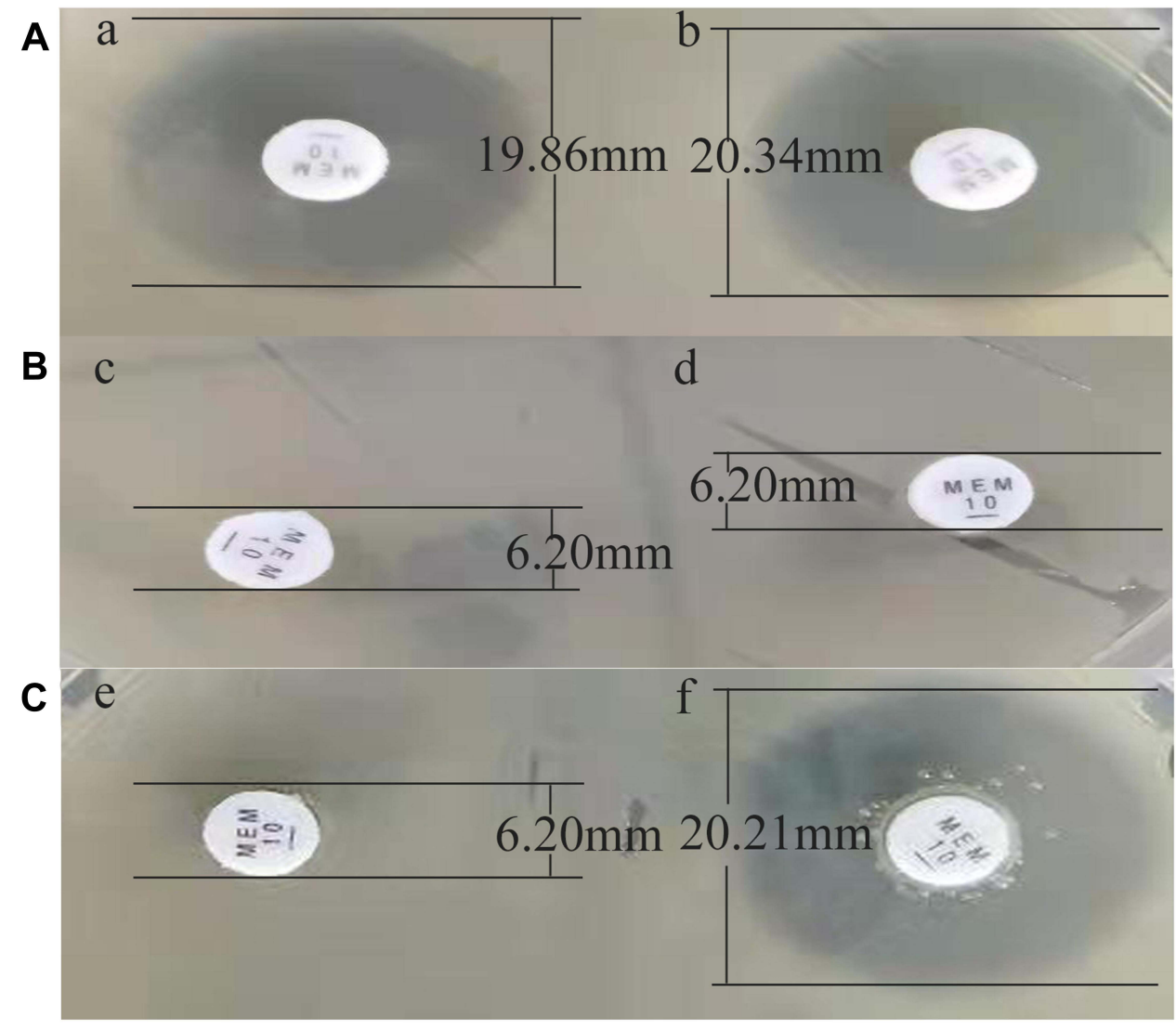

Figure 2 Results of the $\mathrm{mCIM}$ (left) and eCIM (right) tests. (A-C) represent the results of $\mathrm{mCIM}$ and CIM in three different cases respectively. (A) When the transparent inhibition zone is seen around the $\mathrm{mCIM}$ and eCIM discs, the $\mathrm{mCIM}$ (a) test result is negative and eCIM (b) is invalid ( $\mathrm{mCIM}$ diameter of the inhibition zone=19.86 mm and eCIM diameter of the inhibition zone $=20.34 \mathrm{~mm}$ ). (B) Metalloenzymes are produced when the results of $\mathrm{mCIM}$ (c) test are positive (inhibition zone diameter $=6.20 \mathrm{~mm}$ ) and eCIM (d) test are negative (inhibition zone diameter $=6.20 \mathrm{~mm}$ ). (C) When both $\mathrm{mCIM}$ (e) test and eCIM (f) test were positive, serine carbapenemase was produced (diameter of $\mathrm{mCIM}$ inhibition zone $=6.20 \mathrm{~mm}$, diameter of ECIM inhibition zone $=20.21 \mathrm{~mm}$, and the needle scattered colonies in the inhibition zone were negligible). 

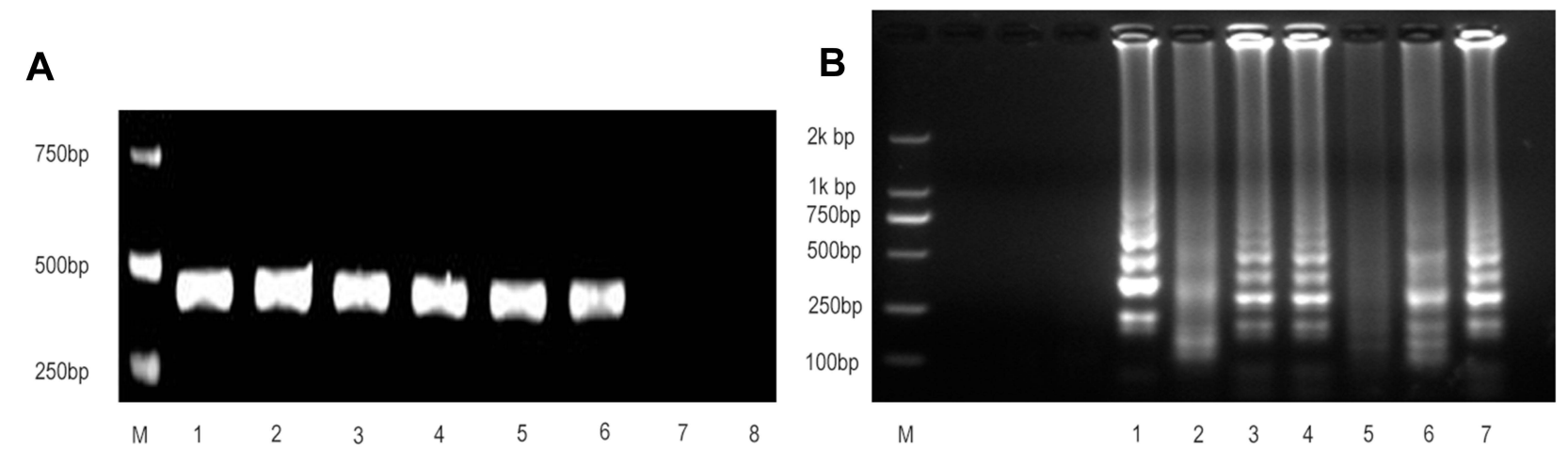

C
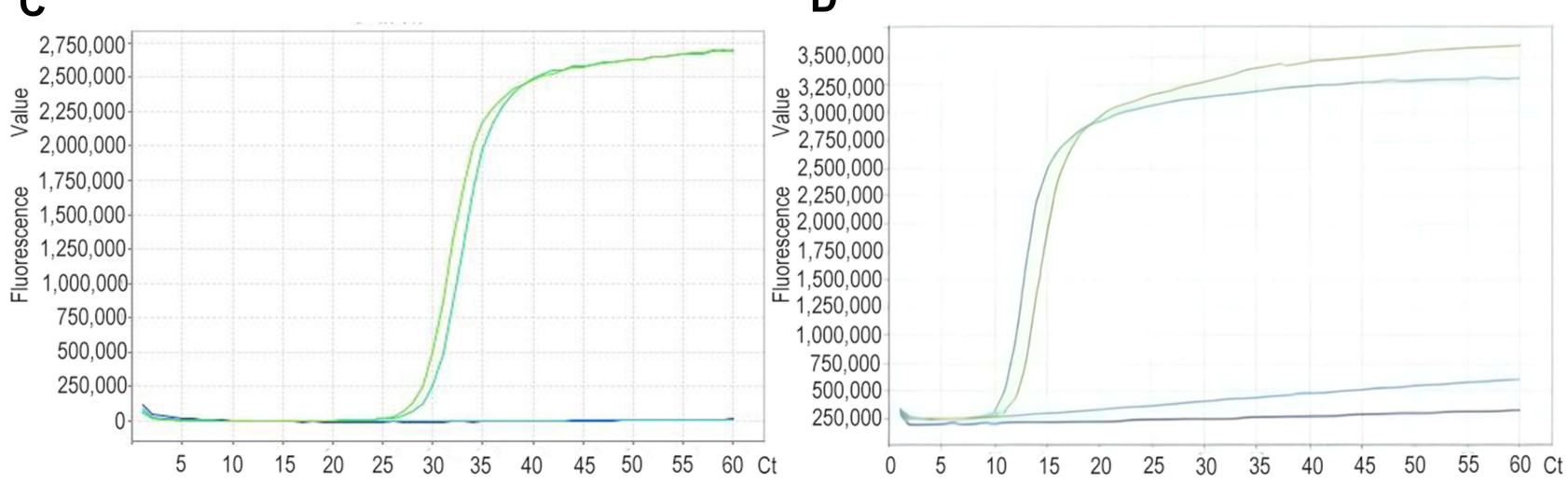

Figure 3 Research design and amplification results: some target genes were successfully amplified by PCR (A) and LAMP (B), and the corresponding PCR and LAMP bands were obtained by agarose gel electrophoresis. For PCR (A), I-6 were positive samples, and 7 and 8 were negative samples. For lamp amplification (B), the step band amplified by the lamp indicates successful amplification (I, 3, 4, 6, 7). The negative samples had no ladder bands (2 and 5). (C and D) respectively represent the signal peaks of the corresponding KPC and NDM genes quantitatively amplified by the light lamp, showing an S-shaped curve, indicating that the gene amplification is successful, and there is no signal peak in the negative sample.

The fluorescence of sequence cleavage was measured and reported every 5 minutes from 0 to $1 \mathrm{~h}$. Fluorescence results and principle of trans-cleavage reaction are shown in Figure 5.

\section{Preparation of Clinical Specimens for Carbapenemase Assay}

A total of 16 clinical strains were collected from patients. These strains were positive or negative for KPC or NDM gene. PCR/LAMP was performed to amplify the target genes. We extracted $100 \mu \mathrm{L}$ DNA from each strain sample as a template for PCR amplification to confirm the presence of KPC and/or NDM enzyme. Before LAMP amplification and DETECTR analysis, the samples were treated at $100{ }^{\circ} \mathrm{C}$ for 8 minutes, followed by centrifugation at $12,000 \times \mathrm{g}$ for 10 minutes. The extracted DNA was used for LAMP analysis. The amplified products were trans-cleaved by Cas12a to observe the results. ${ }^{15}$

\section{Results}

\section{Establishment of CRISPR-Cas I 2a Testing Platform}

The CRISPR-Cas12a rapid detection platform mainly consists of two parts: first, Cas12a-cleaved 5'-FAM-fluorescein -3 'BHQ quencher double-labeled reporter. ${ }^{16}$ The fluorescence value increased significantly on the fluorescence plate reader after Cas12a reaction if the target gene was present. On the contrary, the fluorescence value did not increase significantly. Second, the immunochromatographic strip captured the single-stranded DNA probe cleaved by Cas12a. Therefore, Cas12a reaction is visualized via 5'-FAM-3'-biotin double-labeled reporter, which was cleaved to bind to the specifically designed immunochromatographic strip for capturing single-stranded DNA. ${ }^{17}$ The sequences after LAMP reaction did not contain the target gene if it was not cleaved by Cas12a protein. The FAM fluorescein on the reporter molecule was captured by the antiFAM antibody which was fixed on the first test line on the testing plate to produce a band. The biotin on the cleaved reporter 
A
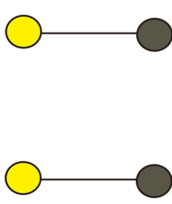

B

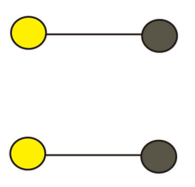

C

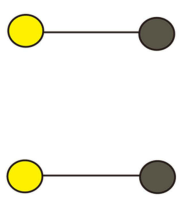

FAM-biotin reporter
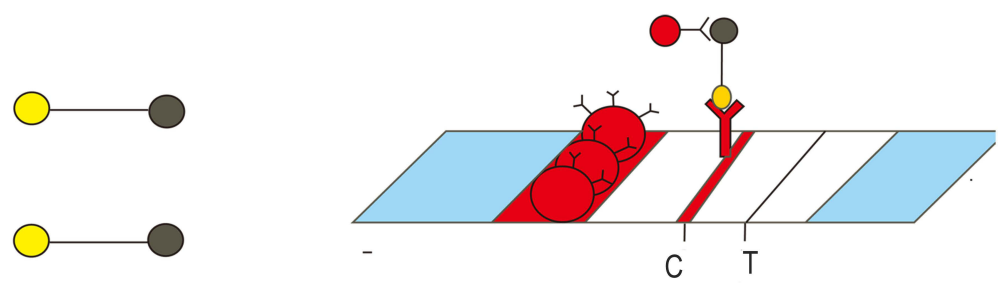

negative
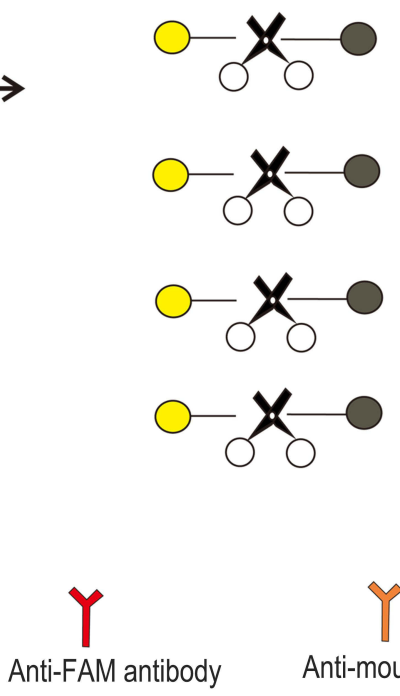

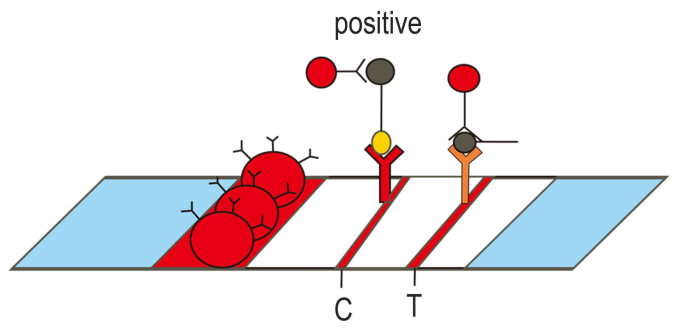

positive

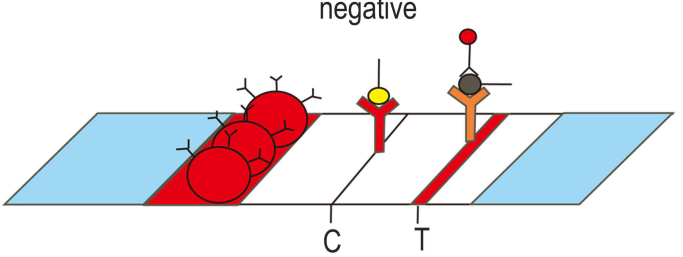

positive

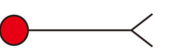

Biotin Antibody Linked to colloidal gold

Figure 4 CRISPR colloidal gold detection results show that: (A) when the fluorescein and biotin double-labeled probe is not cut, it is captured by the anti-FAM antibody in the first row after coupling with the colloidal gold in the diluent. Therefore, the ribbon appears on the control line instead of the test line. (B) When the fluorescein and biotin double-labeled probes were completely cleaved, the anti-mouse antibody on the test line captured the cleaved biotin anti-biotin antibody and colloidal gold complex to display the band, while the control line did not develop color because there was no colloidal gold complex. (C) When the partially cleaved probe was captured, there were colloidal gold-labeled complexes on both the test line and the control line, showing double bands.

molecule was captured by the anti-mouse antibody coupled with colloidal gold on the second test line to produce a band. The bands are visible to the naked eye. The intensity of the test line depends on the concentration of the reporter molecule. When the concentration of the reporter molecule is $50 \mathrm{~nm}$, it is suitable for detection in all assays. ${ }^{18}$

\section{Design and Validation of KPC/NDM Rapid Test Kit}

The main components of CRISPR-Cas system are buffer, crRNA, activator, and Cas12a enzyme. ${ }^{19}$ Crude DNA was used in pilot study. The crude DNA contained sequences of KPC and NDM genes as specific target sequences. Firstly, PCR and LAMP amplification were performed with the crude DNA and clinical bacterial samples to validate the reliability of the method. Secondly, the crude DNA whether amplified or unamplified, were subject to Cas12a cleavage. ${ }^{20}$ Two different reporter molecules (FAM-TTATTTTATT-BHQ; FAM-TTAAAATATT-Biotin) were added. After cleavage, the fluorescence value of the amplicon containing target gene will display a cumulative signal on fluorescence detector. ${ }^{21}$ A red line is visible on the test line of lateral flow strip within 10 minutes. If the target gene is absent, the test line is invisible, and the fluorescence value is not cumulative.

\section{Sensitivity of CRISPR-Cas in Detection of KPC and NDM}

The strains harboring carbapenemase gene screened out by eCIM and $\mathrm{mCIM}$ were adjusted to seven McFarland standards: $3 \times 10 \mathrm{CFU} / \mathrm{mL}, 3 \times 10^{2} \mathrm{CFU} / \mathrm{mL}, 3 \times 10^{3} \mathrm{CFU} / \mathrm{mL}, 3 \times 10^{4} \mathrm{CFU} / \mathrm{mL}, 3 \times 10^{5} \mathrm{CFU} / \mathrm{mL}, 3 \times 10^{6} \mathrm{CFU} / \mathrm{mL}$, and $3 \times 10^{7} \mathrm{CFU} / \mathrm{mL}$ respectively (Figure 6). Bacterial DNA was extracted for RT-LAMP amplification with specific primers. The products generated from LAMP amplification were detected with a lateral flow strip in combination with a biotin probe or fluorescent probe. ${ }^{22}$ The fluorescence signal after Cas12a trans-cleavage was saturated within 20 minutes when 
A

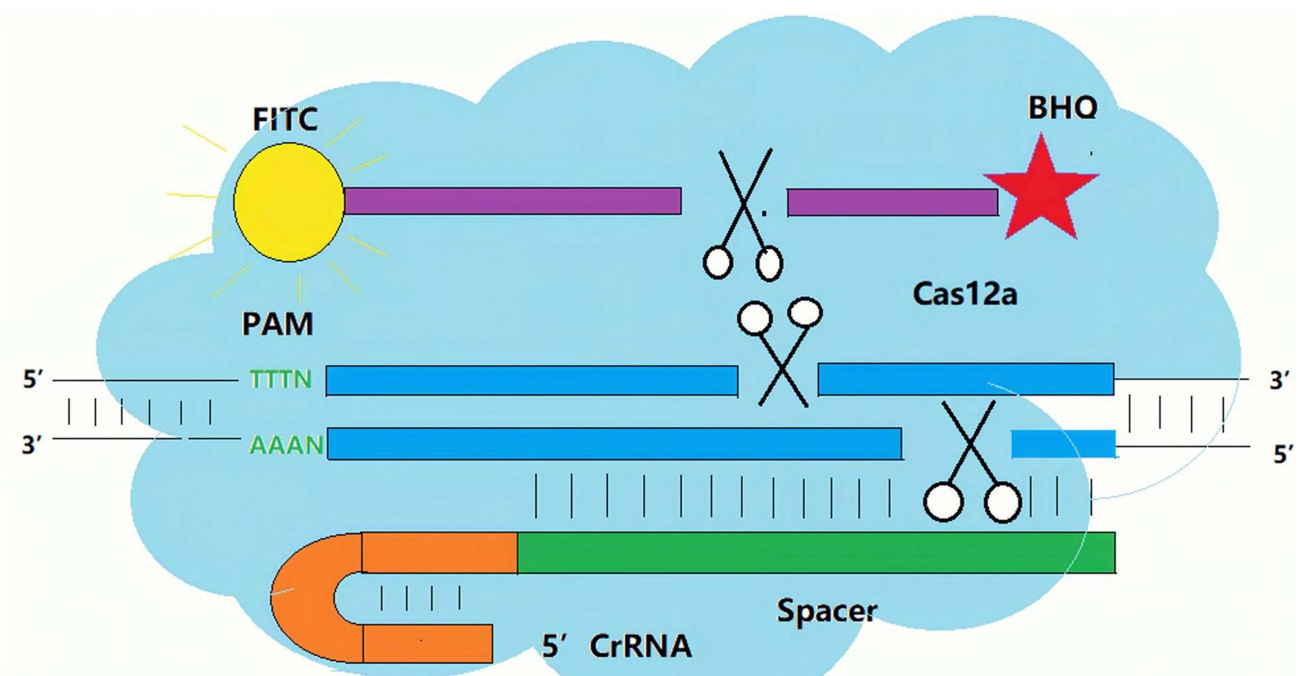

B
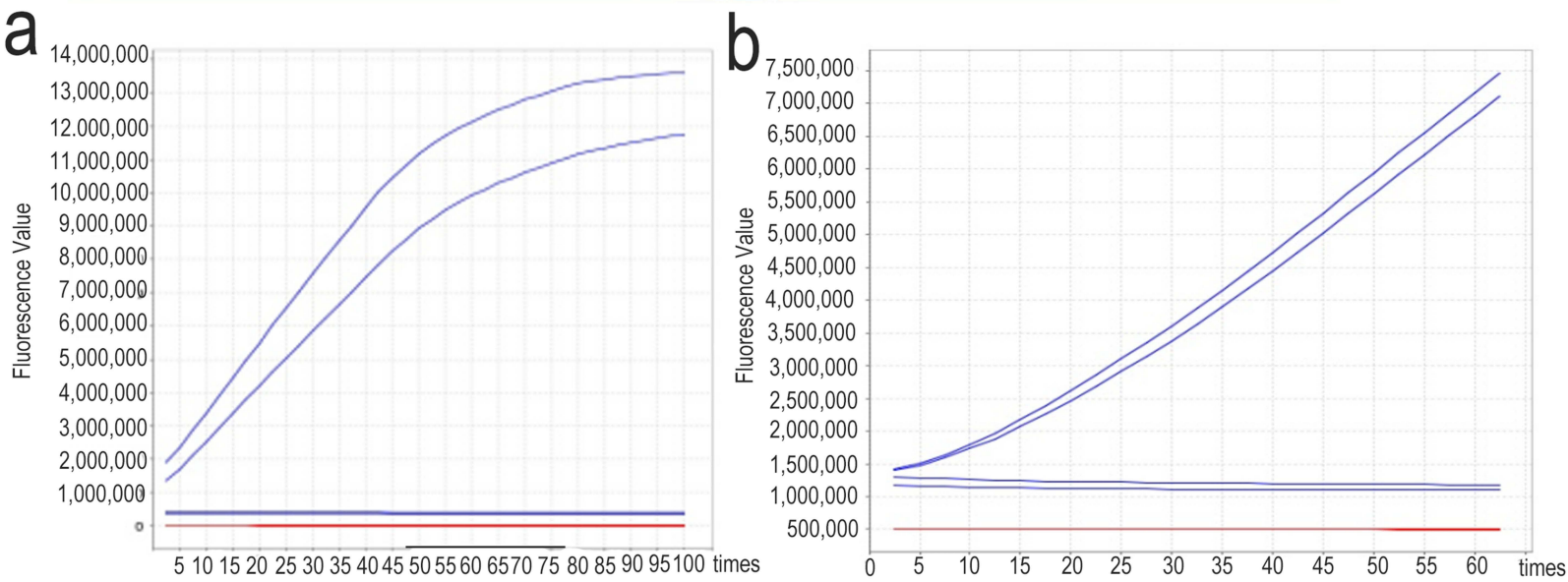

Figure 5 Schematic diagram of Cas 12a trans-cleavage principle and fluorescence-based results: (A) when there are amplified KPC and NDM sequences, gRNA guides Cas I 2a protein, its recognition sequence (green) binds to the target sequence (blue) and cleaves the target sequence, and cleaves the ssDNA probe (the sequence is purple) double labeled with fluorescein (yellow) and quencher (red). (B) When the extinguisher is removed, the fluorescence emitted by the fluorescent probe shows an upward trend. When the target sequence is missing and the single-stranded probe cannot be cleaved, the gRNA cannot recognize the target sequence, and the trans-cleavage of Cas I2a protein does not exist, so no accumulation of fluorescence signal is observed. (a) and (b) represent the fluorescence generated after Cas $12 \mathrm{a}$ cleavage of genes amplified by KPC and NDM enzymes, respectively.

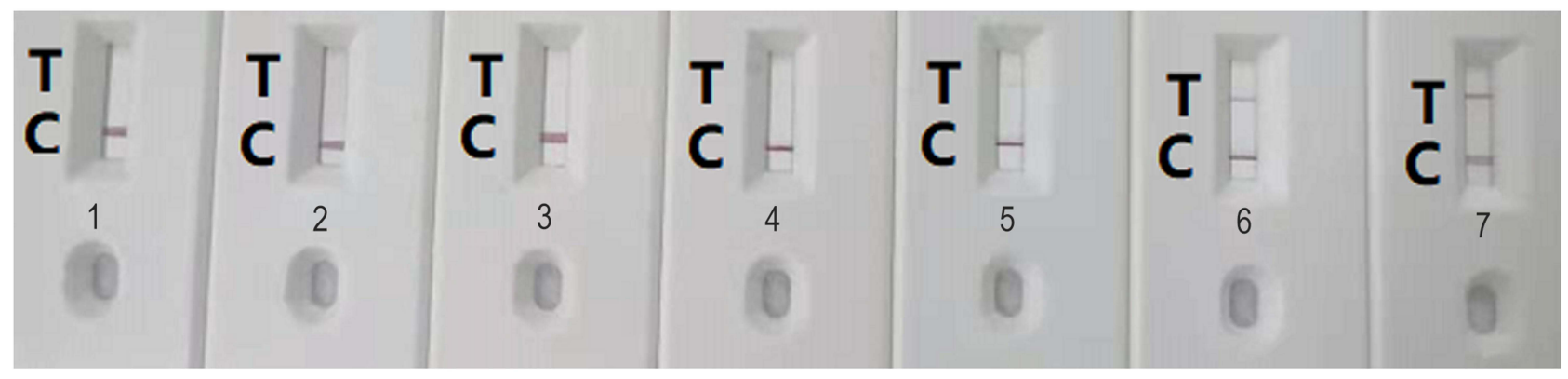

Figure 6 Results of detection after DNA extraction from bacteria of different concentrations (I: $3 \times 10 \mathrm{CFU} / \mathrm{mL}, 2: 3 \times 10^{2} \mathrm{CFU} / \mathrm{mL}, 3: 3 \times 10^{3} \mathrm{CFU} / \mathrm{mL}, 4: 3 \times 10^{4} \mathrm{CFU} / \mathrm{mL}, 5$ : $3 \times 10^{5} \mathrm{CFU} / \mathrm{mL}, 6: 3 \times 10^{6} \mathrm{CFU} / \mathrm{mL}, 7: 3 \times 10^{7} \mathrm{CFU} / \mathrm{mL}$ ). 
the gRNAs of target gene were used to detect RT-LAMP amplified sequences. Biotinylated fluorescein-labeled singlestranded DNA was trans-cleaved by Cas12a and captured by immunochromatographic strip to produce the target band. ${ }^{23}$

\section{Validation of DETECTR for KPC/NDM Detection in Clinical Samples}

Sensitivity test of colony number of carbapenem producing bacteria. Before DETECTR assay, the selected strains $(1 \mathrm{~mL}$ per sample) were heated to $100^{\circ} \mathrm{C}$ for 5 minutes, followed by centrifugation at $12,000 \times \mathrm{g}$ for 10 minutes. The supernatant was taken as template. The KPC- or NDM-producing samples showed a detectable test line as the result of reporter transcleavage. The KPC/NDM carbapenemase-nonproducing samples did not display visible test lines within an hour for LAMP and $\sim 2 \mathrm{~h}$ for PCR-based Cas12a assays. ${ }^{24}$ As illustrated in Figure 3C, PCR/LAMP amplification also corroborated with the DETECTR assay results. Overall, unlike previously reported methods, such as stand-alone PCR and LAMP, and other CRISPR-based assays, which require expensive materials such as electrophoretic, fluorescence apparatus, and monoclonal antibodies, our method was quick and convenient with inexpensive readout. ${ }^{25}$ Hence, it is applicable for on-site analysis of nucleic acids in samples with greatly enhanced detection efficiency and convenience. ${ }^{26}$

\section{Specificity of DETECTR in Detection of KPC/NDM}

Different clinical samples were used to evaluate and validate the specificity of DETECTR assay. Ten $\mu \mathrm{L}$ of Cas12a transcleaved KPC/NDM carbapenemase-containing samples applied to the lateral flow strip assay. For the target genecontaining samples, a strong signal was observed at the test lines, indicating cleavage of the reporter; whereas, in the absent of target gene, no visible test line was detected, the band was observed only on the control line, highlighting no trans-cleavage of the reporter. These findings indicate that our lateral flow strip has very high specificity. Clinical sample test shows that six strains of Klebsiella pneumoniae containing KPC enzyme were detected. All 8 strains of Klebsiella pneumoniae containing NDM enzyme showed test lines, and other samples of Klebsiella pneumoniae without these two enzymes did not show test lines (Figure 7).

\section{Discussion}

Currently, the treatment options for carbapenemase-producing Enterobacterales are limited in clinical practice. The commonly used therapies are carbapenems, tigecycline, and polymyxins, as monotherapy or in combination. These last resorts cannot realize the desired efficacy in some cases. Recently, new drugs are developed by taking advantages of lactamase inhibitors, such as ceftazidime/avibactam and aztreonam/avibactam. It has been reported that ceftazidime/ avibactam can inhibit KPC-type serine lactamases, while aztreonam/avibactam can suppress New Delhi metallo- $\beta$ lactamase (NDM). Antimicrobial combinations have made some difference in the in vitro susceptibility test, but the results are usually inconsistent with clinical findings. ${ }^{27}$ The species and number of pathogens, and the plasma protein binding rate of antibiotics are different in different patient population. The breakpoints for interpreting the results of in vitro susceptibility testing do not consider the pharmacokinetic factors. ${ }^{28}$ Therefore, rapid lab detection and genotyping of carbapenem-resistant strains is very important for the antimicrobial treatment and outcomes of CRE infections.

These resistant strains are currently detected by disk diffusion method, E-test, broth microdilution method, and automated antimicrobial susceptibility testing systems. The phenotypic assay of carbapenem-resistant organisms includes Carba NP, mCIM and eCIM assay, and enhancement of antimicrobial activity by $\beta$-lactamase inhibitor. Genotype-based assays including PCR, multiplex PCR, nested PCR, and RT-PCR are combined with microfluidic technology to analyze the genotypes or identify the strains via sequencing analysis. ${ }^{29}$ These techniques are not only dependent upon expensive lab instruments, but also time-consuming and cumbersome. They are not available in ordinary laboratories. For such resistant strains, it is urgent for us to establish a more rapid, simple, and accurate detection method to address the "bottleneck problem" about rapid detection of resistant bacteria. ${ }^{30}$ Timely and accurate etiological diagnosis is necessary for better informing early, quick, and precision antimicrobial treatment.

We designed and developed a new technique based on CRISPR-Cas12a and combined with LAMP to meet the needs for rapid and affordable diagnostic tools. ${ }^{31}$ This technique enables us to couple with a lateral flow immunochromatographic strip to develop a rapid test for detecting carbapenemases in clinical samples within 30-40 minutes. There are some evident advantages for this new tool. Firstly, only simple equipment is required for 


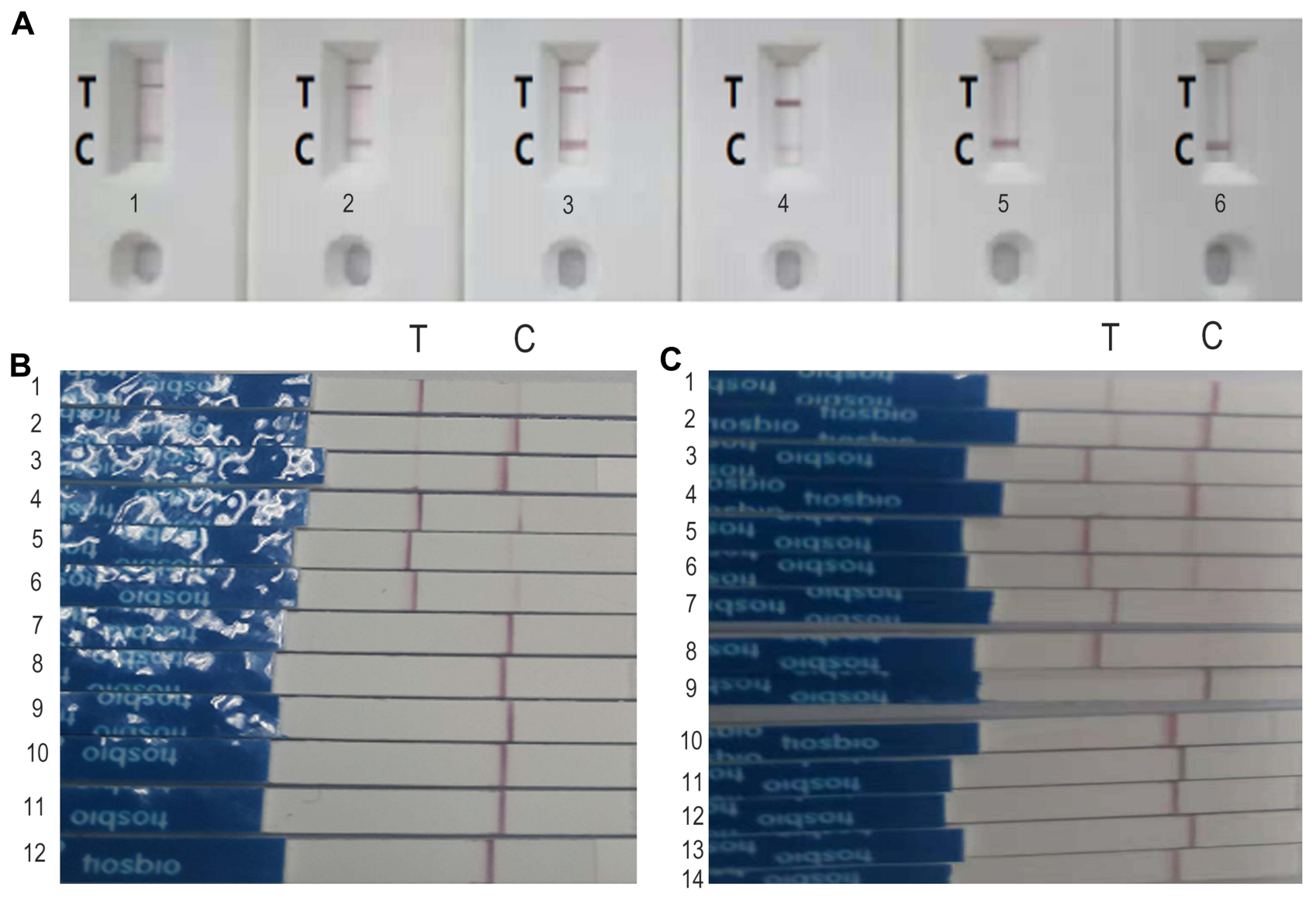

Figure $\mathbf{7}$ We tested the samples with CRISPR immunochromatographic strips with card (A) and immunochromatographic strips without card (B and C). The positive sample has two detection lines and the negative sample has one detection line because the FAM fluorescein biotin probe cut by Cas I $2 \mathrm{a}$ protein is coupled with colloidal gold and captured by the anti-mouse antibody on the test line, the probe not cut by Cas I 2a protein is captured by the anti-FAM antibody on the control line. (A) No. I and 2 contain KPC gene, No. 3 and 4 contain NDM gene, and there are corresponding bands on the test line. No. 5 and 6 contain neither KPC nor NDM gene. So the test results are negative and only the control lines are displayed. (B) Twelve strains were detected, of which 6 strains containing KPC (No. I-6) were positive and the other 6 strains (No. 7-12) were negative without KPC gene. (C) Fourteen strains were detected, of which eight strains (No. I-8) contained NDM gene and six strains (No. 9-14) did not contain NDM gene.

LAMP amplification. The sensitivity is higher than conventional PCR. The CRISPR-Cas12a technique can specifically trans-cleave the reporter to prevent the effect of false-positive results of LAMP. The results therefore appear on the lateral flow strip visible to the naked eye. Secondly, in contrast to the conventional cumbersome and timeconsuming laboratory assays, the newly developed method in this study greatly reduced the turnaround time. ${ }^{32}$ It is not necessary for the operators to receive repeated training. The new tool enables us to accurately determine the genotypes of resistant genes. Finally, a rapid testing platform can be set up based on this technique. The platform is applicable in other pathogens of great public health concerns, such as SARS-CoV-2, human papillomavirus, and new virus of Bunyaviridae. It is easily modified by changing LAMP primers and crRNA alone. This assay can still provide more diagnostic results under resource-constrained environment. ${ }^{33}$ Some limitations are also found for this technique despite the above strengths. For example, dimer may form in the process of LAMP amplification. CRISPR-Cas-based assay can only detect known DNA sequences. So, it is not applicable for unknown genotypes. In addition, the current test kit must be stored at $-20{ }^{\circ} \mathrm{C} .{ }^{34}$ The storage method and conditions should be modified and improved to ensure longer shelf-life at room temperature for large scale and extensive clinical use. We are just getting started to develop this new laboratory assay. ${ }^{35}$ It is reasonable to believe that the technology is promising for quickly identifying refractory pathogens. ${ }^{36}$ The technique is leading a biotechnology revolution in large-scale population screening to control outbreak infection. ${ }^{37}$ 


\section{Ethics Approval and Consent to Participate}

This study involved anonymous use of redundant, abandoned patient sputum, which is part of the standard treatment agreements with patients in our hospital. This research does not affect patients' health and privacy. All procedures performed in studies involving human participants were in accordance with the ethical standards of the medical ethics committee of the Fourth Affiliated Hospital of Anhui Medical University (reference number: LISC20210802) and with the 1964 Helsinki Declaration and its later amendments or comparable ethical standards.

\section{Author Contributions}

All authors made a significant contribution to the work reported, whether that is in the conception, study design, execution, acquisition of data, analysis and interpretation, or in all these areas; took part in drafting, revising, or critically reviewing the article; gave final approval of the version to be published; have agreed on the journal to which the article has been submitted; and agree to be accountable for all aspects of the work.

\section{Funding}

This work was funded through a grant from Anhui Provincial Department of Education for university cooperative research and public health collaborative innovation project in Anhui Province in 2020 (Grant No. GXXT-2020-016) and a grant from Anhui Provincial Health Commission for key scientific research projects in 2021 (Grant No. AHWJ2021a011).

\section{Disclosure}

The authors declare that they have no competing interests.

\section{References}

1. Broughton JP, Deng X, Yu G, et al. CRISPR-Cas12-based detection of SARS-CoV-2. Nat Biotechnol. 2020;38(7):870-874. doi:10.1038/s41587020-0513-4

2. Lahiri S, Venkataraman R, Jagan A, et al. Evaluation of LAMP-based assays for carbapenemase genes. J Med Microbiol. 2019;68(10):1431-1437. doi:10.1099/jmm.0.001050

3. Nakano R, Nakano A, Ishii Y, et al. Rapid detection of the Klebsiella pneumoniae carbapenemase (KPC) gene by loop-mediated isothermal amplification (LAMP). J Infect Chemother. 2015;21(3):202-206. doi:10.1016/j.jiac.2014.11.010

4. Solanki R, Vanjari L, Ede N, et al. Evaluation of LAMP assay using phenotypic tests and conventional PCR for detection of bla NDM-1 and bla KPC genes among carbapenem-resistant clinical Gram-negative isolates. J Med Microbiol. 2013;62(Pt 10):1540-1544. doi:10.1099/ jmm.0.059907-0

5. Srisrattakarn A, Lulitanond A, Wilailuckana C, et al. Rapid and simple identification of carbapenemase genes, bla NDM, bla OXA-48, bla VIM, bla IMP-14 and bla KPC groups, in Gram-negative bacilli by in-house loop-mediated isothermal amplification with hydroxynaphthol blue dye. World J Microbiol Biotechnol. 2017;33(7):130. doi:10.1007/s11274-017-2295-5

6. Zalas-Więcek P, Gospodarek-Komkowska E, Smalczewska A. Rapid detection of genes encoding extended-spectrum beta-lactamase and carbapenemase in clinical Escherichia coli isolates with eazyplex SuperBug CRE system. Microb Drug Resist. 2020;26(10):1245-1249. doi:10.1089/ mdr.2019.0311

7. Baek YH, Cheon HS, Park SJ, et al. Rapid and sensitive portable molecular diagnosis of SFTS virus using Reverse Transcriptional Loop-Mediated Isothermal Amplification (RT-LAMP). J Microbiol Biotechnol. 2018;28(11):1928-1936. doi:10.4014/jmb.1806.06016

8. Rödel J, Egerer R, Suleyman A, et al. Use of the variplex ${ }^{\mathrm{TM}}$ SARS-CoV-2 RT-LAMP as a rapid molecular assay to complement RT-PCR for COVID-19 diagnosis. J Clin Virol. 2020;132:104616. doi:10.1016/j.jcv.2020.104616

9. Kashir J, Yaqinuddin A. Loop mediated isothermal amplification (LAMP) assays as a rapid diagnostic for COVID-19. Med Hypotheses. 2020;141:109786. doi:10.1016/j.mehy.2020.109786

10. Augustine R, Hasan A, Das S, et al. Loop-mediated isothermal amplification (LAMP): a rapid, sensitive, specific, and cost-effective point-of-care test for coronaviruses in the context of COVID-19 pandemic. Biology (Basel). 2020;9(8):182.

11. Zhang H, Xu Y, Fohlerova Z, et al. LAMP-on-a-chip: revising microfluidic platforms for loop-mediated DNA amplification. Trends Analyt Chem. 2019;113:44-53. doi:10.1016/j.trac.2019.01.015

12. Yao R, Liu D, Jia X, et al. CRISPR-Cas9/Cas12a biotechnology and application in bacteria. Synth Syst Biotechnol. 2018;3(3):135-149. doi:10.1016/j.synbio.2018.09.004

13. Chen JS, Ma E, Harrington LB, et al. CRISPR-Cas12a target binding unleashes indiscriminate single-stranded DNase activity. Science. 2018;360 (6387):436-439. doi:10.1126/science.aar6245

14. Murugan K, Seetharam AS, Severin AJ, et al. CRISPR-Cas12a has widespread off-target and dsDNA-nicking effects. J Biol Chem. 2020;295 (17):5538-5553. doi:10.1074/jbc.RA120.012933

15. Manghwar H, Lindsey K, Zhang X, et al. CRISPR/Cas System: recent advances and future prospects for genome editing. Trends Plant Sci. 2019;24 (12):1102-1125. doi:10.1016/j.tplants.2019.09.006 
16. Kim H, Lee WJ, Oh Y, et al. Enhancement of target specificity of CRISPR-Cas12a by using a chimeric DNA-RNA guide. Nucleic Acids Res. 2020;48(15):8601-8616. doi:10.1093/nar/gkaa605

17. Liang M, Li Z, Wang W, et al. A CRISPR-Cas12a-derived biosensing platform for the highly sensitive detection of diverse small molecules. Nat Commun. 2019;10(1):3672. doi:10.1038/s41467-019-11648-1

18. Bai J, Lin H, Li H, et al. Cas12a-based on-site and rapid nucleic acid detection of African swine fever. Front Microbiol. 2019;10:2830. doi:10.3389/ fmicb.2019.02830

19. Swartjes T, Staals RHJ, van der Oost J. Editor's cut: DNA cleavage by CRISPR RNA-guided nucleases Cas9 and Cas12a. Biochem Soc Trans. 2020;48(1):207-219. doi:10.1042/BST20190563

20. Zhao Y, Boeke JD. CRISPR-Cas12a system in fission yeast for multiplex genomic editing and CRISPR interference. Nucleic Acids Res. 2020;48 (10):5788-5798. doi:10.1093/nar/gkaa329

21. Dai Y, Somoza RA, Wang L, et al. Exploring the trans-cleavage activity of CRISPR-Cas12a (cpf1) for the development of a universal electrochemical biosensor. Angew Chem Int Ed Engl. 2019;58(48):17399-17405. doi:10.1002/anie.201910772

22. Wang B, Wang R, Wang D, et al. Cas12aVDet: a CRISPR/Cas12a-based platform for rapid and visual nucleic acid detection. Anal Chem. 2019;91 (19):12156-12161. doi:10.1021/acs.analchem.9b01526

23. Stella S, Mesa P, Thomsen J, et al. Conformational activation promotes CRISPR-Cas12a catalysis and resetting of the endonuclease activity. Cell. 2018;175(7):1856-1871.e21. doi:10.1016/j.cell.2018.10.045

24. Breinig M, Schweitzer AY, Herianto AM, et al. Multiplexed orthogonal genome editing and transcriptional activation by Cas12a. Nat Methods. 2019;16(1):51-54. doi:10.1038/s41592-018-0262-1

25. Lee K, Zhang Y, Kleinstiver BP, et al. Activities and specificities of CRISPR/Cas9 and Cas12a nucleases for targeted mutagenesis in maize. Plant Biotechnol J. 2019;17(2):362-372. doi:10.1111/pbi.12982

26. Sun Y, Liu H, Shen Y, et al. Cas12a-activated universal field-deployable detectors for bacterial diagnostics. ACS Omega. 2020;5(24):14814-14821. doi:10.1021/acsomega.0c01911

27. Ding X, Yin K, Li Z, et al. All-in-one dual CRISPR-Cas12a (AIOD-CRISPR) Assay: a case for rapid, ultrasensitive and visual detection of novel coronavirus SARS-CoV-2 and HIV virus. bioRxiv. 2020;21(03):998724.

28. Smith CW, Nandu N, Kachwala MJ, et al. Probing CRISPR-Cas12a nuclease activity using double-stranded DNA-templated fluorescent substrates. Biochemistry. 2020;59(15):1474-1481. doi:10.1021/acs.biochem.0c00140

29. Mukama O, Yuan T, He Z, et al. A high fidelity CRISPR/Cas12a based lateral flow biosensor for the detection of HPV16 and HPV18. Sens Actuators B Chem. 2020;316:128119. doi:10.1016/j.snb.2020.128119

30. Yuan T, Mukama O, Li Z, et al. A rapid and sensitive CRISPR/Cas12a based lateral flow biosensor for the detection of Epstein-Barr virus. Analyst. 2020;145(19):6388-6394. doi:10.1039/D0AN00663G

31. Curti LA, Federico PB, Guillermo DR, et al. CRISPR-based platform for carbapenemases and emerging viruses detection using Cas12a (Cpf1) effector nuclease. Emerg Microbes Infect. 2020;9(1):1140-1148. doi:10.1080/22221751.2020.1763857

32. Bathoorn E, Tsioutis C, da Silva Voorham JM, et al. Emergence of pan-resistance in KPC-2 carbapenemase-producing Klebsiella pneumoniae in Crete, Greece: a close call. J Antimicrob Chemother. 2016;71(5):1207-1212. doi:10.1093/jac/dkv467

33. Chen L, Mathema B, Chavda KD, et al. Carbapenemase-producing Klebsiella pneumoniae: molecular and genetic decoding. Trends Microbiol. 2014;22(12):686-696. doi:10.1016/j.tim.2014.09.003

34. Esra DC, Nilüfer A. Klebsiella pneumoniae: characteristics of carbapenem resistance and virulence factors. Acta Biochim Pol. 2015;62(4):867-874. doi:10.18388/abp.2015 1148

35. da Silva RM, Traebert J, Galato D. Klebsiella pneumoniae carbapenemase (KPC)-producing Klebsiella pneumoniae: a review of epidemiological and clinical aspects. Expert Opin Biol Ther. 2012;12(6):663-671. doi:10.1517/14712598.2012.681369

36. Arnold RS, Thom KA, Sharma S, Phillips M, Kristie johnson J, Morgan DJ. Emergence of Klebsiella pneumoniae carbapenemase-producing bacteria. South Med J. 2011;104(1):40-45. doi:10.1097/SMJ.0b013e3181fd7d5a

37. Mustafa MI, Makhawi AM. SHERLOCK and DETECTR: CRISPR-Cas systems as potential rapid diagnostic tools for emerging infectious diseases. J Clin Microbiol. 2021;59(3):e00745-20. doi:10.1128/JCM.00745-20

Infection and Drug Resistance

\section{Publish your work in this journal}

Infection and Drug Resistance is an international, peer-reviewed open-access journal that focuses on the optimal treatment of infection (bacterial, fungal and viral) and the development and institution of preventive strategies to minimize the development and spread of resistance. The journal is specifically concerned with the epidemiology of antibiotic resistance and the mechanisms of resistance development and diffusion in both hospitals and the community. The manuscript management system is completely online and includes a very quick and fair peer-review system, which is all easy to use. Visit http://www.dovepress.com/testimonials.php to read real quotes from published authors.

Submit your manuscript here: https://www.dovepress.com/infection-and-drug-resistance-journal 